\title{
A convex hull' characterization
}

\section{Franco Fineschi, Giovanni Quaranta}

Department DISAG, University of Siena, Italy

\section{Email address:}

fineschi@unisi.it (F. Fineschi), quaranta@unisi.it (G. Quaranta)

\section{To cite this article:}

Franco Fineschi, Giovanni Quaranta. A Convex Hull' Characterization. Pure and Applied Mathematics Journal. Vol. 3, No. 2, 2014, pp. 40-48. doi: 10.11648/j.pamj.20140302.13

\begin{abstract}
Conditions so that a vector belongs to a convex hull are obtained. Multilinear convex functions are considered. If these maps are defined on a convex set, it is obtained the algebraic expression. As an application, infinite games, with linear convex payoff, are studied.
\end{abstract}

Keywords: Convex, Linear, Game

\section{Introduction}

Some properties of the class of the $k$-linear convex functions are considered. These maps seem to be the natural extention of $k$-linear functions if the domain is bounded. Given a linear convex mapping, defined on a body, it is possible to extend its definition if an argument is outside of the domain. This property enables us to find the analytical form of a $k$-linear convex function $\phi: X^{k} \rightarrow Y$, if $X$ is a convex set which contains the null vector.

An arbitrary element of a convex set can be represented in three different ways using finite sequence of elements of the set; these representations are obtained by the following theorems: the Shapley-Folkman lemma, Carathéodory's theorem and Fenchel-Bunt' theorem.

For example by the last theorem, for any element sof a convex, connected set $X$, a sequence of $n$ elements of $X$ exists such that $s$ is expressed by a convex combination of the sequence. In spite of this, it is well known that an arbitrary convex set has not a "convex basis", that is, it is impossible to find an unique sequence, with finite number of elements of the set, which span, by linear convex combinations, any other. In Algebra it is proved that $\mathfrak{R}^{n}$ is free over the standard basis, this implies some properties, one of these is that an arbitrary sequence of a module $A$ may be written as a linear combination of the vectors $t\left(e_{1}\right), \ldots, t\left(e_{n}\right)$ for an unique morphism $t: \mathfrak{R}^{n} \rightarrow A$. This concept of free set is now moved to convex sets by linear convex functions. One result obtained in this paper is that a convex combination for an element of a convex, connected set $A$ is expressed by a unique convex combination of vectors $\phi\left(e_{1}\right), \ldots, \phi\left(e_{n}\right)$ for a linear convex function $\phi$, that is, any element $a$ of $A$ determines a $\phi$ such that $\sum \xi_{i} \phi\left(e_{i}\right)=a$.

Conditions are obtained in order that a vector belongs to a convex hull if it is spanned by a finite set. The conditions give new expression to the convex hull.

It is known, see [7] and [8], that two persons, infinite, symmetric games, with a compact, convex set as strategy space and a linear convex function as payoff, have solutions. In the last section we obtain the form of the payoff of these games.

\section{Free Convex Sets}

Let $S$ be a set of vectors in $\Re^{n}$, with the null vector $\underline{0} \in S$, then denote by $X=\cos$ the convex hull of $S$. Suppose $k \leq n$ the maximal number of linearly independent vectors in $X$ and $x_{1}, \ldots, x_{k}$ be a set of linearly independent vectors of $X$.

A $k$-linear convex mapping $\phi: X^{k} \rightarrow A$, for $a_{i} \in X$, is defined by

$\phi\left(a_{1}, \ldots, a_{i}, \ldots, a_{k}\right)=\phi\left(a_{1}, \ldots, \sum_{i=1}^{r} \lambda_{i} b_{i}, \ldots, a_{k}\right)=\sum_{i=1}^{r} \lambda_{i} \phi\left(a_{1}, \ldots, b_{i}, \ldots, a_{k}\right)$

where $\lambda_{i} \geq 0$ and $\sum_{i=1}^{r} \lambda_{i}=1, b_{i} \in \mathfrak{R}^{n}$.

Example 2.1Consider the function

$$
f(x, y)=2 x y \quad 0 \leq x \leq a, \quad 0 \leq y \leq b
$$

Let $\frac{a}{2}<x_{1}<x_{2}<a$, then $f\left(x_{1}, y\right)=2 x_{1} y$ and $f\left(x_{2}, y\right)=2 x_{2} y$, even if $f\left(x_{1}+x_{2}, y\right)$ does not exist, so 
$f(x, y)$ is not a bilinear function. Whereas, for $0<\lambda<1$,

$$
\begin{gathered}
f\left(\lambda x_{1}+(1-\lambda) x_{2}, y\right)=2\left(\lambda x_{1}+(1-\lambda) x_{2}\right) y \\
=2 \lambda x_{1} y+2(1-\lambda) x_{2} y \\
=\lambda f\left(x_{1}, y\right)+(1-\lambda) f\left(x_{2}, y\right)
\end{gathered}
$$

that is, $f(x, y)$ is a convex linear function of each variable separately.

The following is a known basic statement

Proposition 2.1 Let $\phi: X^{k} \rightarrow \Re^{m}$ be a k-linear convex mapping and $X$ a convex subset of $\mathfrak{R}^{n}$. The image $\phi\left(X^{k}\right)$ is convex in $\mathfrak{R}^{m}$.

Proof. The image under $\phi$ of every convex combination of elements of $X$ is a convex combination in $\mathfrak{R}^{m}$.

A point of a convex hull has three known representations by sequences of elements of the same convex set.

a)The first representation is founded on the Shapley-Folkman lemma, see [1], [2] and [10].Consider a finite family $S_{i}, i=1, \ldots, k$ of subset of $\mathfrak{K}^{n}$, the Minkowsky sum $s=\sum_{i=1}^{k} s_{i}$ is defined by element-wise addiction of vectors

$$
s=\left\{s_{1}+\cdots+s_{k}: s_{i} \in S_{i}\right\}
$$

The Minkowsky sum has an immediate property: for any nonempty finite family of sets $S_{i}$ of $\Re^{n} \operatorname{co}\left(\sum S_{i}\right)=\sum \operatorname{coS} S_{i}$.

So if $s \in \sum \operatorname{coS} S_{i}$ and $s=s_{1}+\cdots+s_{k}$ with $s_{i} \in \operatorname{coS}_{i}$. The expression of sby $s_{1}+\cdots+s_{k}$ depends on the same point $s$. For the representation $s=s_{1}+\cdots+s_{k}$, in the space $\mathfrak{R}^{n}$, holds

Lemma (Shapley-Folkman) If the number $k$ of $S_{i}$ is greater than the dimension $n$ of the space, the point $s$ has the expression

$$
s=\sum_{1 \leq i \leq n} s_{i}+\sum_{n+1 \leq j \leq k} s_{j}
$$

where $s_{i} \in \operatorname{coS}_{i}$ and $s_{j} \in S_{j}$.

In other words $s \in \sum_{1 \leq i \leq n} \operatorname{coS}_{i}+\sum_{n+1 \leq j \leq k} S_{j}$.

b)By the Carathéodory's theorem, any $s \in \operatorname{coS} \subset \mathfrak{R}^{n}$ may be represented as a convex combination of $n+1$ elements of $S$.

c)In particular, if $S$ is connected, the Fenchel-Bunt' theorem, see [3] and [8], proves that any $s \in \cos$ can be expressed as a convex combination of $n$ elements of $S$. Thesethree representations of an element do not establish the existence of a "convex basis" for $\cos$, because the $n$ elements may depend on the particular s to be computed, see [6]. Nevertheless, the next definition asserts the condition in order that a convex set is free. For a definition of a free module on a subset, see [4].

In the following we present a different representation for elements of convex set based on the definition of free convex set.

Definition 2.1 Let $K$ be a subset of a convex set $X$ in $\mathfrak{R}^{n}$ and let $j: K \rightarrow X$ be the insertion of $K$ in $X$. Denote by $A$ a subset of $\mathfrak{R}^{m}$, then $X$ is free over $K$ if, for every function $f: K \rightarrow A$, an unique linear convex mapping $\phi: X \rightarrow A$ exists such that $\phi \circ j=f$.

Example 2.2. Let $K=\{a, b\} \subset \mathfrak{R}^{2}$ be a subset and coK its convex hull. We want to prove that coK is free over $K$, i.e., for every $f: K \rightarrow A$ an unique linear convex function $\phi: c o K \rightarrow A$ exists such that $\phi \circ j=f$.

Unicity. The condition $\phi \circ j=f$ means that $\phi(a)=$ $f(a)$ and $\phi(b)=f(b)$. Then for every $f: K \rightarrow A$, we have to prove the unicity of $\phi: c o K \rightarrow A$ such that $\phi(a)=f(a)$ and $\phi(b)=f(b)$. For any $k=\lambda_{1} a+\lambda_{2} b \in \operatorname{coK}$ and for every linear convex function $\phi$ holds $\phi(k)=\phi\left(\lambda_{1} a+\right.$ $\left.\lambda_{2} b\right)=\lambda_{1} \phi(a)+\lambda_{2} \phi(b)=\lambda_{1} f(a)+\lambda_{2} f(b)$ so $\phi$ is univocally defined.

Existence. The function $\phi$, defined by $\phi\left(\lambda_{1} a+\lambda_{2} b\right)=$ $\lambda_{1} f(a)+\lambda_{2} f(b)$, is linear convex, in fact for every $x=\left(\xi_{1} a+\xi_{2} b\right) \in \operatorname{coK}$

$$
\phi\left(\xi_{1} a+\xi_{2} b\right)=\xi_{1} f(a)+\xi_{2} f(b)=\xi_{1} \phi(a)+\xi_{2} \phi(b)
$$

Explicitly the expression of the unique linear convex $\phi$ is given by

$$
\begin{gathered}
\phi(k)=\phi\left(\lambda_{1} a+\lambda_{2} b\right)=\lambda_{1} f(a)+\lambda_{2} f(b)= \\
\lambda_{1}\left(c_{11}, c_{21}, c_{31}\right)+\lambda_{2}\left(c_{12}, c_{22}, c_{32}\right)= \\
=\left(\begin{array}{ll}
c_{11} & c_{12} \\
c_{21} & c_{22} \\
c_{31} & c_{32}
\end{array}\right)\left(\begin{array}{l}
\lambda_{1} \\
\lambda_{2}
\end{array}\right)=C_{\phi}\left(\begin{array}{l}
\lambda_{1} \\
\lambda_{2}
\end{array}\right)
\end{gathered}
$$

Where it is supposed $f(a)=\left(c_{11}, c_{21}, c_{31}\right), f(b)=$ $\left(c_{12}, c_{22}, c_{32}\right)$ and $f(a), f(b) \in A \subseteq \mathfrak{R}^{3}$,for any $k=$ $\left(\lambda_{1} a+\lambda_{2} b\right) \in \mathrm{coK}$ with fixed $a$ and $b$. Then, $\phi$ is the matrix $C_{\phi}$.

The underlying property of the example above will be proved in a more general setting.

Some properties of the mapping $\phi: X^{k} \rightarrow A$ follow.

Since $\quad X \quad$ is convex, $\forall a_{i} \in X, 0<\alpha<1$, $\alpha a_{i}+(1-\alpha) \underline{0}=\alpha a_{i} \in X$. In particular $\alpha a_{i} \in X$. Moreover

$$
\begin{aligned}
& \Phi\left(a_{1}, \ldots, \alpha a_{i}, \ldots, a_{k}\right)=\Phi\left(a_{1}, \ldots, \alpha a_{i}+(1-\alpha) \underline{0}, \ldots, a_{k}\right) \\
& =\alpha \Phi\left(a_{1}, \ldots, a_{i}, \ldots, a_{k}\right)+(1-\alpha) \Phi\left(a_{1}, \ldots, \underline{0}, \ldots, a_{k}\right)
\end{aligned}
$$

supposed $a_{i}=a_{j}$ and $\Phi$ skewsymmetric it follows

$$
\Phi\left(a_{1}, \ldots, \alpha a_{i}, \ldots, a_{j}, \ldots, a_{k}\right)=(1-\alpha) \Phi\left(a_{1}, \ldots, \underline{0}, \ldots, a_{j}, \ldots, a_{k}\right)
$$

comparing 1 and 2, it follows $\Phi$ skewsymmetric and

$$
\begin{gathered}
\alpha \Phi\left(a_{1}, \ldots, a_{i}, \ldots, a_{j}, \ldots, a_{k}\right)=\Phi\left(a_{1}, \ldots, \alpha a_{i}, \ldots, a_{j}, \ldots, a_{k}\right) \\
-\Phi\left(a_{1}, \ldots, \alpha a_{j}, \ldots, a_{j}, \ldots, a_{k}\right)
\end{gathered}
$$


Let $\beta \in \mathfrak{R}^{+}$and $m \in N-\{0\}$ such that $0<\frac{\beta}{m}<1$, $\forall a_{i} \in X$, by the (3) it holds

$$
\begin{gathered}
\beta \phi\left(a_{1}, \ldots, a_{i}, \ldots, a_{j}, \ldots, a_{k}\right)=m \phi\left(a_{1}, \ldots, \frac{\beta}{m} a_{i}, \ldots, a_{j}, \ldots, a_{k}\right) \\
-m \phi\left(a_{1}, \ldots, \frac{\beta}{m} a_{j}, \ldots, a_{j}, \ldots, a_{k}\right)
\end{gathered}
$$

Proposition $2.2 \quad$ Let $\lambda \in \mathfrak{R}^{+}\left(\lambda \in \mathfrak{R}^{-}\right) \quad$ and $\lambda x \in X, \lambda>|1|$, then $\alpha x \in X(-\alpha x \in X)$, for $0<\alpha<1$.

Proof. If $0<\beta<1 \quad$ satisfies $\alpha=\beta \cdot \lambda$, then $\alpha x=\beta(\lambda x)+(1-\beta) \underline{0}$, so $\alpha x \in X$. If $-\alpha=\beta \cdot \lambda$ then $-\alpha x=\beta(\lambda x)+(1-\beta) \underline{0}$ and $-\alpha x \in X$.

The next proposition extends the (1) and defines a linear convex mapping if its argument is outside the body

Proposition 2.3 Let $x_{1}, \ldots, x_{i}, \ldots, x_{k}$ be vectors in $X$ and $\delta \in \mathfrak{R}$, then a linear convex mapping $\phi: X^{k} \rightarrow A$ satisfies

$$
\begin{aligned}
& \phi\left(x_{1}, \ldots, \delta x_{i}, \ldots, x_{k}\right)=\delta \phi\left(x_{1}, \ldots, x_{i}, \ldots, x_{k}\right) \\
& +(1-\delta) \phi\left(x_{1}, \ldots, \underline{0}, \ldots, x_{k}\right)
\end{aligned}
$$

Proof. Let $0<\lambda, \mu, v<1$ such that $\delta=\frac{1}{\lambda} \mu-\frac{1-\lambda}{\lambda} v$ and $\lambda|\delta|<1$. It is always possible to determinate such numbers $\lambda, \mu, v$. Indeed, if $\lambda=\frac{1}{\alpha}<1$, the relation $\delta=\frac{1}{\lambda} \mu-\frac{1-\lambda}{\lambda} v$ becames $\delta=\alpha \mu-(\alpha-1) \nu$, then, for example suppose $v=\frac{1}{2}$, for $\alpha>2|\delta|+1$, it holds $0<\mu=\frac{2 \delta+\alpha-1}{2 \alpha}<1$.

Then, by the Proposition 2.2 , the vectors $\mu x_{i}, \nu x_{i}$ are in $X$ and the mapping $\phi$ is defined on them.

$$
\begin{aligned}
& \left.\phi\left(x_{1}, \ldots, \mu x_{i}, \ldots, x_{k}\right)=\phi\left(x_{1}, \ldots,(\lambda \delta+(1-\lambda) v)\right) x_{i}, \ldots, x_{k}\right) \\
& =\lambda \phi\left(x_{1}, \ldots, \delta x_{i}, \ldots, x_{k}\right)+(1-\lambda) \phi\left(x_{1}, \ldots, v x_{i}, \ldots, x_{k}\right) \text { that is } \\
& \lambda \phi\left(x_{1}, \ldots, \delta x_{i}, \ldots, x_{k}\right)=\phi\left(x_{1}, \ldots, \mu x_{i}, \ldots, x_{k}\right) \\
& -(1-\lambda) \phi\left(x_{1}, \ldots, v x_{i}, \ldots, x_{k}\right)
\end{aligned}
$$

by the (1)

$$
\begin{aligned}
& \phi\left(x_{1}, \ldots, \delta x_{i}, \ldots, x_{k}\right)=\frac{1}{\lambda} \phi\left(x_{1}, \ldots, \mu x_{i}+(1-\mu) \underline{0}, \ldots, x_{k}\right) \\
& -\frac{(1-\lambda)}{\lambda} \phi\left(x_{1}, \ldots, \nu x_{i}+(1-v) \underline{0}, \ldots, x_{k}\right) \\
& =\frac{\mu}{\lambda} \phi\left(x_{1}, \ldots, x_{i}, \ldots, x_{k}\right)+\frac{(1-\mu)}{\lambda} \phi\left(x_{1}, \ldots, \underline{0}, \ldots, x_{k}\right) \\
& -\frac{(1-\lambda) v}{\lambda} \phi\left(x_{1}, \ldots, x_{i}, \ldots, x_{k}\right)
\end{aligned}
$$

$$
\begin{aligned}
& -\frac{(1-\lambda)(1-v)}{\lambda} \phi\left(x_{1}, \ldots, \underline{0}, \ldots, x_{k}\right) \\
& =\left(\frac{\mu}{\lambda}-\frac{(1-\lambda) v}{\lambda}\right) \phi\left(x_{1}, \ldots, x_{i}, \ldots, x_{k}\right) \\
& +\left(\frac{(1-\mu)}{\lambda}-\frac{(1-\lambda)(1-v)}{\lambda}\right) \phi\left(x_{1}, \ldots, \underline{0}, \ldots, x_{k}\right) \\
& =\delta \phi\left(x_{1}, \ldots, x_{i}, \ldots, x_{k}\right)+(1-\delta) \phi\left(x_{1}, \ldots, \underline{0}, \ldots, x_{k}\right)
\end{aligned}
$$

Theorem 2.1 Let $\phi: X^{k} \rightarrow Y$ be a k-linear convex function and $X$ a convex set with $\underline{0} \in X$, then $\phi\left(a_{1}, \ldots, a_{k}\right)$ may be expressed by a linear combination of $\phi\left(x_{j_{1}}, \ldots, x_{j_{k}}\right)$, where $x_{j_{i}} \in X$ span the vectors $a_{i} \in X$.

Proof. The nonnull vectors $a_{1}, \ldots, a_{k}$ in $X$ may be written as

$a_{i}=\gamma_{1 i} x_{1}+\cdots+\gamma_{k i} x_{k}=\left(\gamma_{1}, \ldots, \gamma_{k}\right)$

$=k\left\|a_{i}\right\| b_{i}$

$=k\left\|a_{i}\right\|\left(\frac{1}{k} \frac{\gamma_{1 i}}{\left\|a_{i}\right\|} x_{1}+\cdots+\frac{1}{k} \frac{\gamma_{k i}}{\left\|a_{i}\right\|} x_{k}\right)$

$=k\left\|a_{i}\right\| \sum_{j=1}^{k} \frac{1}{k} \frac{\gamma_{j i}}{\left\|a_{i}\right\|} x_{j}$

$=k\left\|a_{i}\right\| \sum_{j=1}^{k} \frac{1}{k} b_{j i} x_{j} \quad k \in N-\{0\}$

where $-1 \leq b_{i j}=\frac{\gamma_{j i}}{\left\|a_{i}\right\|} \leq 1, i, j=1, \ldots, k$.

By $k$ applications of the Proposition 2.3 to the arguments of $\phi$, it follows

$\phi\left(a_{1}, \ldots, a_{k}\right)=\phi\left(k\left\|a_{1}\right\| b_{1}, \ldots, k\left\|a_{k}\right\| b_{k}\right)$

$=k\left\|a_{1}\right\| \phi\left(b_{1}, a_{2}, \ldots, a_{k}\right)+\left(1-k\left\|a_{1}\right\|\right) \phi\left(\underline{0}, a_{2}, \ldots, a_{k}\right)$

$=k^{2}\left\|a_{1}\right\|\left\|a_{2}\right\| \phi\left(b_{1}, b_{2}, a_{3}, \ldots, a_{k}\right)$

$+k\left\|a_{1}\right\|\left(1-k\left\|a_{2}\right\|\right) \phi\left(b_{1}, \underline{0}, a_{3}, \ldots, a_{k}\right)$

$+k\left\|a_{2}\right\|\left(1-k\left\|a_{1}\right\|\right) \phi\left(\underline{0}, b_{2}, a_{3}, \ldots, a_{k}\right)$

$+\left(1-k\left\|a_{1}\right\|\right)\left(1-k\left\|a_{2}\right\|\right) \phi\left(\underline{0}, \underline{0}, a_{3}, \ldots, a_{k}\right)$

$=k^{k}\left\|a_{1}\right\| \cdots\left\|a_{k}\right\| \phi\left(b_{1}, \ldots, b_{k}\right)$

$+\sum_{i=1}^{k} k^{k-1}\left\|a_{1}\right\| \cdots\left\|a_{i-1}\right\|\left\|a_{i+1}\right\| \cdots\left\|a_{k}\right\|\left(1-k\left\|a_{i}\right\|\right)$.

$\cdot \phi\left(b_{1}, \ldots, b_{i}=\underline{0}, \ldots, b_{k}\right)+\cdots$ 


$$
\begin{aligned}
& +\sum_{i_{1}<\cdots<i_{r}} k^{k-r}\left\|a_{j_{1}}\right\| \cdots\left\|a_{j_{k-r}}\right\|\left(1-k\left\|a_{i_{1}}\right\|\right) \cdots\left(1-k\left\|a_{i_{r}}\right\|\right) . \\
& \cdot \phi\left(b_{1}, \ldots, b_{i_{1}}=\underline{0}, \ldots, b_{i_{r}}=\underline{0}, \ldots, b_{k}\right)+\cdots \\
& +\left(1-k\left\|a_{1}\right\|\right)\left(1-k\left\|a_{2}\right\|\right) \cdots\left(1-k\left\|a_{k}\right\|\right) \phi(\underline{0}, \underline{0}, \ldots, \underline{0})
\end{aligned}
$$

where $i_{1}, \ldots, i_{r}$ is the complement of $j_{i}, \ldots, j_{k-r}$ with respect to the set $1, \ldots, k$ and the sum in (6) is over all the subsets $i_{1}, \ldots, i_{r}$

Now use the convex linearity on all the vectors $b_{i}$

$$
\begin{aligned}
& \phi\left(b_{1}, \ldots, b_{k}\right)=\phi\left(\sum_{j=1}^{k} \frac{1}{k} b_{j 1} x_{j}, \ldots, \sum_{j=1}^{k} \frac{1}{k} b_{j k} x_{j}\right) \\
& =\frac{1}{k} \sum_{i=1}^{k} \phi\left(b_{i 1} x_{i}, b_{2}, \ldots, b_{k}\right) \\
& =\ldots \quad \ldots \quad \ldots \quad \ldots \\
& =\frac{1}{k^{k}} \sum_{j_{1}} \ldots \sum_{j_{k}} \phi\left(b_{j_{1} 1} x_{j_{1}}, \ldots, b_{j_{k} k} x_{j_{k}}\right)
\end{aligned}
$$

where, by the (1)

$$
\begin{aligned}
& \phi\left(b_{j_{1} 1} x_{j_{1}}, \ldots, b_{j_{k} k} x_{j_{k}}\right)=b_{j_{1} 1} \phi\left(x_{j_{1}}, \ldots, b_{j_{k} k} x_{j_{k}}\right) \\
& +\left(1-b_{j_{1} 1}\right) \phi\left(\underline{0}, b_{j_{2} 2} x_{j_{2}}, \ldots, b_{j_{k} k} x_{j_{k}}\right) \\
& =b_{j_{1} 1}, \ldots, b_{j_{k} k} \phi\left(x_{j_{1}}, \ldots, x_{j_{k}}\right) \\
& +\sum_{j_{i}=j_{1}}^{j_{k}} b_{j_{1}}, \ldots, b_{j_{i-1} i-1}, b_{j_{i+1} i+1}, \ldots, b_{j_{k} k}\left(1-b_{j_{i}}\right) . \\
& \phi \phi\left(x_{j_{1}}, \ldots, x_{j_{i}}=\underline{0}, \ldots, x_{j_{k}}\right)+\cdots \\
& +\sum_{i_{1}<\cdots<i_{r}} b_{s_{1}} \cdots b_{s_{k-r}}\left(1-b_{i_{1}}\right) \cdots\left(1-b_{i_{r}}\right) . \\
& \phi\left(x_{j_{1}}, \ldots, x_{i_{1}}=\underline{0}, \ldots, x_{i_{r}}=\underline{0}, \ldots, x_{j_{k}}\right)+\cdots \\
& +\left(1-b_{j_{1}}\right) \cdots\left(1-b_{j_{k}}\right) \phi(\underline{0}, \underline{0}, \ldots, \underline{0})
\end{aligned}
$$

where $i_{1}, \ldots, i_{r}$ is the complement of $s_{1}, \ldots, s_{k-r}$ with respect to the set $1, \ldots, k$ and the sum in (7) is over all the subsets $i_{1}, \ldots, i_{r}$.

So $\phi\left(a_{1}, \ldots, a_{k}\right)$ is obtained by a linear expression in $\phi\left(x_{j_{1}}, \ldots, x_{j_{k}}\right)$, where some $x_{j_{i}}$ can be the null vector $\underline{0}$.

In the n-dimensional vector space $\mathfrak{R}^{n}$, denote by $S_{n}$ the convex hull of the vectors $\left\{e_{1}, \ldots, e_{n}\right\}$ of the standard basis. $S_{n}$ is a compact, connected, convex set and its elements may be expressed by convex combinations of the unit vectors $\left\{e_{1}, \ldots, e_{n}\right\}$.

Theorem 2.2 The set $S_{n}$ is free over the standard basis $\left\{e_{1}, \ldots, e_{n}\right\}$ of $\mathfrak{R}^{n}$.

Proof. Let $K=\left\{e_{1}, \ldots, e_{n}\right\}$, the condition $\phi \circ j=f$ implies $\phi\left(e_{i}\right)=f\left(e_{i}\right)=a_{i}$ and the function $\phi$ is defined by

$$
\phi\left(\xi_{1} e_{1}+\cdots+\xi_{e_{n}}\right)=\sum \xi_{i} a_{i} \quad \xi_{i} \in \Re^{+}, \sum \xi_{i}=1, a_{i} \in A
$$

Uniqueness. If $\phi$ is a linear convex function with $\phi\left(e_{i}\right)=a_{i}$, then, for any $k \in S_{n}$

$$
\phi(k)=\phi\left(\xi_{1} e_{1}+\cdots+\xi_{n} e_{n}\right)=\sum \xi_{i} \phi\left(e_{i}\right)=\sum \xi_{i} a_{i} \quad \sum \xi_{i}=1
$$

so $\phi$ is unique.

Existence. $\phi(k)=\sum \xi_{i} a_{i}$ defines a function $\phi: S_{n} \rightarrow A$. Let us prove that $\phi$ is a linear convex function. $\forall k \in S_{n}$ let $k=\xi_{1} e_{1}+\cdots+\xi_{n} e_{n}, \sum \xi_{i}=1$, then

$$
\phi(k)=\phi\left(\xi_{1} e_{1}+\cdots+\xi_{n} e_{n}\right)=\sum \xi_{i} a_{i}=\xi_{1} \phi\left(e_{1}\right)+\cdots+\xi_{n} \phi\left(e_{n}\right)
$$

By the Fenchel-Bunt' theorem, any element $a$ of a compact, connected, convex set $A$ is expressed as a convex combination of the sequence $a_{1}, \ldots, a_{n}$ of vectors of $A$, that is $a=\xi_{1} a_{1}+\cdots+\xi_{n} a_{n}$. By the theorem 2.2 exists an unique linear convex function $\phi$ such that

$$
\phi\left(\xi_{1} e_{1}+\cdots+\xi_{n} e_{n}\right)=\sum \xi_{i} a_{i}=a=\sum \xi_{i} \phi\left(e_{i}\right)
$$

so, any element $a \in A$ may be expressed as a convex combination of the vectors $\phi\left(e_{i}\right), \ldots, \phi\left(e_{n}\right)$. In other words, any $a \in A$ determines a linear convex function $\phi$ such that $\sum \xi_{i} \phi\left(e_{i}\right)=a$.

Example 2.3 Let $A$ be a convex, connected set in $\mathfrak{R}^{2}$. If $a=\xi_{1} a_{1}+\xi_{2} a_{2}, \xi_{i} \geq 0, \sum \xi_{i}=1, a_{i}=\left(a_{i 1}, a_{i 2}\right)$ is an element of $A$, then, by the theorem 2.2, it follows

$$
a=\phi\left(\xi_{1} e_{1}+\xi_{2} e_{2}\right)=\xi_{1} a_{1}+\xi_{2} a_{2}=\xi_{1} \phi\left(e_{1}\right)+\xi_{2} \phi\left(e_{2}\right)
$$

where $\phi: S_{2} \rightarrow A$ is linear convex. This implies $\phi\left(e_{1}\right)=a_{1}, \phi\left(e_{2}\right)=a_{2}$, and so

$$
\phi(x)=\left(\begin{array}{ll}
a_{11} & a_{21} \\
a_{12} & a_{22}
\end{array}\right)(x) \quad x \in S_{2}
$$

\section{A Convex Hull' Characterization}

Let $a_{1}, \ldots, a_{m}$ be a finite set of vectors in $\mathfrak{R}^{m}$, then its convex hull is defined by 


$$
\operatorname{co}\left\{a_{1}, \ldots, a_{m}\right\}=\left\{\sum_{i=1}^{m} \alpha_{i} a_{i}, \alpha=\left(\alpha_{1}, \ldots, \alpha_{m}\right) \in \Sigma_{m}\right\}
$$

where $\Sigma_{m}$ is the unit simplex in $\mathfrak{R}^{m}$. The next proposition lays down a condition so that a vector belongs to a convex hull.

Theorem 3.1 Let co $\left\{a_{1}, \ldots, a_{m}\right\}$ be a convex hull where $A=\left(a_{1}, \ldots, a_{m}\right)$ is a matrix with $|A| \neq 0$, then $b \in \operatorname{co}\left\{a_{1}, \ldots, a_{m}\right\}$ iff

(i).

$$
\left|\begin{array}{cccc}
1 & 1 & \ldots & 1 \\
b_{1} & a_{11} & \ldots & a_{1 m} \\
\ldots & \ldots & \ldots & \ldots \\
b_{m} & a_{m 1} & \ldots & a_{m m}
\end{array}\right|=0
$$

\section{(ii). The determinants}

$\left|b, a_{2}, \ldots, a_{m}\right|,\left|a_{1}, b, a_{3}, \ldots, a_{m}\right|, \ldots,\left|a_{1}, a_{2}, \ldots, a_{m-1}, b\right|,|A|$

have the same sign.

Proof. Suppose $b \in \operatorname{co}\left\{a_{1}, \ldots, a_{m}\right\}$, then $\sum_{i=1}^{m} \alpha_{i} a_{i}=b$ for $\alpha=\left(\alpha_{1}, \ldots, \alpha_{m}\right) \in \Sigma_{m}$, that is, $A \alpha=b$. Then

$$
\alpha_{j}=\frac{\left|a_{1}, \ldots, a_{j-1}, b, a_{j+1}, \ldots, a_{m}\right|}{|A|}
$$

is the solution of the system $A \alpha=b$. Impose $\sum_{j=1}^{m} a_{j}=1$, we obtain

$$
\left|b, a_{2}, \ldots, a_{m}\right|+\left|a_{1}, b, a_{3}, \ldots, a_{m}\right|+\ldots+\left|a_{1}, a_{2}, \ldots, a_{m-1}, b\right|=|A|
$$

and so the (i).

Conversely, if (i) and (ii) hold, moving backward, we get $\alpha$ such that $A \alpha=b$, then $b \in \operatorname{co}\left\{a_{1}, \ldots, a_{m}\right\}$.

Next step is the extension of the Theorem 3.1 to a convex hull given by the vectors $a_{1}, \ldots, a_{n}$, in $\mathfrak{R}^{m}$, with $n>m$ and $r(A)=r$. For this purpose it is necessary to introduce some generalizations of elementary definitions in linear algebra. For more details see [5].

The multindex $I_{r}^{n}$ of length $r$ is defined by

$$
I_{r}^{n}=\left\{\left(i_{1}, \ldots, i_{r}\right): 1 \leq i_{1}<\ldots<i_{r} \leq n\right\}
$$

besides we define, for a fixed natural number $k$,

$$
\begin{aligned}
& \left(I_{r}^{n}\right)_{k}=\left\{\left(i_{1}, \ldots i_{p} \ldots, i_{r}\right): 1 \leq i_{1}<\ldots<i_{p}=k<\ldots<i_{r} \leq n,\right. \\
& \text { where } 1 \leq k \leq n\}
\end{aligned}
$$

Let $\mathrm{F}$ be a field with $\operatorname{char}(\mathrm{F}) \neq 2$, then, for an arbitrary matrix, consider the linear form in the parameters $\lambda_{\beta}^{\alpha} \in \mathrm{F}$ given by
Definition 3.1 Let $\Delta_{r}: \mathrm{F}^{m \times n} \rightarrow\left(\mathrm{F}\left(\begin{array}{l}m \\ r\end{array}\right)\left(\begin{array}{l}n \\ r\end{array}\right)\right)^{*}$ be a map defined by

$$
\Delta_{r} A=\sum_{\alpha, \beta}\left|A_{\beta}^{\alpha}\right| \lambda_{\beta}^{\alpha}, \quad \alpha \in I_{r}^{m}, \beta \in I_{r}^{n} .
$$

If $m=r=r(A)$, then $\left|A_{\beta}^{\alpha}\right|$, in $\Delta_{m} A$, are the Plücker coordinates of the subspace spanned by the rows of $A$.

Example 3.1 For the matrix $A=\left(\begin{array}{lll}a_{11} & a_{12} & a_{13} \\ a_{21} & a_{22} & a_{23}\end{array}\right)$, with $r(A)=2$

$$
\Delta_{2} A=\left|\begin{array}{lll}
a_{11} & a_{12} & a_{13} \\
a_{21} & a_{22} & a_{23}
\end{array}\right|
$$

$$
=\left|\begin{array}{ll}
a_{11} & a_{12} \\
a_{21} & a_{22}
\end{array}\right| \lambda_{12}^{12}+\left|\begin{array}{ll}
a_{11} & a_{13} \\
a_{21} & a_{23}
\end{array}\right| \lambda_{13}^{12}+\left|\begin{array}{ll}
a_{12} & a_{13} \\
a_{22} & a_{23}
\end{array}\right| \lambda_{23}^{12}
$$

The definition of cofactor $\alpha_{i j}$ can be extended to an arbitrary matrix.

Definition 3.2 Let $A \in \mathrm{F}^{m \times n}$ be a matrix with $r(A)=r$, the cofactor $\alpha_{i j}^{r}$ of the entry $a_{i j}$ of $A$, for $m<n$, is defined by

$$
\alpha_{i j}^{r}=\left|\begin{array}{ccccc}
a_{11} & \ldots & \ldots & \ldots & a_{1 n} \\
& \ldots & \ldots & \ldots & \\
0 & \ldots & 1 & \ldots & 0 \\
& \ldots & \ldots & \ldots & \\
a_{m 1} & \ldots & \ldots & \ldots & a_{m n}
\end{array}\right|^{i}
$$

$=\Delta_{r} A: a_{i x}=0$ for $\mathrm{x}=1, \ldots, \mathrm{j}-1, \mathrm{j}+1, \ldots, \mathrm{n}, \mathrm{a}_{\mathrm{ij}}=1$ and $\lambda_{\beta}^{\alpha} \neq 0$ only if $\mathrm{i} \in \alpha$

$\sum_{i_{1} \ldots i_{1} \ldots i_{r} j_{1} \ldots j \ldots j_{r}} \lambda_{j_{1} \ldots j \ldots j_{r}}^{i_{1} \ldots i_{r} \ldots i_{r}}\left|\left(A_{j_{1} \ldots j \ldots j_{r}}^{i_{1} \ldots i_{1} \ldots i_{r}}\right)_{i-\text { th row }=e_{j}^{\prime}}\right|$

where in the last determinant the i-th row is $e_{j}^{\prime}=(0, \ldots, 1, \ldots, 0)$, i.e. the $j$-th unit vector. The sum is over all $i_{1}, \ldots, i, \ldots, i_{r} \in\left(I_{r}^{m}\right)_{i}$ and $j_{1}, \ldots, j, \ldots, j_{r} \in\left(I_{r}^{n}\right)_{j}$.

Similarly, for $m>n$,

$$
\alpha_{i j}^{r}=\left|\begin{array}{ccccc}
a_{11} & \ldots & 0 & \ldots & a_{1 n} \\
\ldots & \ldots & \ldots & \ldots & \ldots \\
a_{i 1} & \ldots & 1 & \ldots & a_{i n} \\
\ldots & \ldots & \ldots & \ldots & \ldots \\
a_{m 1} & \ldots & 0 & \ldots & a_{m n}
\end{array}\right|_{\underline{j}}
$$

Example 3.2 For $A \in \mathrm{F}^{3 \times 4}$ and $r(A)=2$ 


$$
\begin{aligned}
& \alpha_{23}^{r}=\left|\begin{array}{cccc}
a_{11} & a_{12} & a_{13} & a_{14} \\
0 & 0 & 1 & 0 \\
a_{31} & a_{32} & a_{33} & a_{34}
\end{array}\right|^{2}=\left|\begin{array}{cccc}
a_{11} & a_{12} & a_{13} & a_{14} \\
0 & 0 & 1 & 0
\end{array}\right|^{12} \\
& +\left|\begin{array}{cccc}
0 & 0 & 1 & 0 \\
a_{31} & a_{32} & a_{33} & a_{34}
\end{array}\right|^{23}=\left(\begin{array}{cc}
a_{11} & a_{13} \\
0 & 1
\end{array}\left|\lambda_{13}^{12}+\right| \begin{array}{cc}
a_{12} & a_{13} \\
0 & 1
\end{array} \mid \lambda_{23}^{12}\right. \\
& \left.+\left|\begin{array}{cc}
a_{13} & a_{14} \\
1 & 0
\end{array}\right| \lambda_{34}^{12}\right)+\left(\begin{array}{cc}
0 & 1 \\
a_{31} & a_{33}
\end{array}\left|\lambda_{13}^{23}+\right| \begin{array}{cc}
0 & 1 \\
a_{32} & a_{33}
\end{array}\left|\lambda_{23}^{23}+\right| \begin{array}{cc}
1 & 0 \\
a_{33} & a_{34}
\end{array} \mid \lambda_{34}^{23}\right)
\end{aligned}
$$

As in the example, it will be useful to write the numbers of the rows (columns) involved in the expansion of the cofactor like apexes (indexes).

Let $C=\left(\alpha_{i j}^{r}\right)$ the r-cofactor matrix of $A$. The transpose of $C$, i.e. the r-adjoints of $A$ will be denoted by $A^{a d}$.

Theorem 3.2 Any consistent linear system $A x=b, b \neq 0$, where $A \in \mathrm{F}^{m \times n}, \quad r(A)=r$, has as set of solutions

$$
x_{j}=\frac{\alpha_{1 j}^{r} b_{1}+\cdots+\alpha_{m j}^{r} b_{m}}{\Delta_{r} A} \quad j=1, \ldots, n
$$

for any $\lambda_{\beta}^{\alpha} \in \mathrm{F}$ such that $r\left(A^{a d}\right) \geq r$.

Proof. $\left(\Delta_{r} A\right)^{-1} A^{a d}$ are the (1)-inverses of $A$, then the solutions of $A x=b$ are $x=\left(\Delta_{r} A\right)^{-1} A^{a d} b$.

The Theorem 3.2 is an evident Cramer's rule generalization.

All that is necessary to the next proposition is now ready.

Theorem 3.3 Let co $\left\{a_{1}, \ldots, a_{n}\right\}$ be a convex hull where $A=\left(a_{1}, \ldots, a_{n}\right)$ is a matrix with $r(A)=m, \quad a_{i} \neq 0 i=1, \ldots, n$, then $b \in \operatorname{co}\left\{a_{1}, \ldots, a_{n}\right\}$ iff

(i)

$$
\gamma_{11}^{m}+b_{1} \gamma_{21}^{m}+\cdots+b_{m} \gamma_{m+1,1}^{m}=0
$$

where the cofactors $\gamma_{i j}^{m}$ are obtained by the matrix

$$
C=\left(\begin{array}{cccc}
1 & 1 & \ldots & 1 \\
b_{1} & a_{11} & \ldots & a_{1 m} \\
\ldots & \ldots & \ldots & \ldots \\
b_{m} & a_{m 1} & \ldots & a_{m m}
\end{array}\right)
$$

(ii) The sums $\alpha_{1 j}^{m} b_{1}+\cdots+\alpha_{m j}^{m} b_{m}$, with $j=1, \ldots, n$, have the same sign of $\Delta_{m} A$.

Proof. Suppose $b \in \operatorname{co}\left\{a_{1}, \ldots, a_{n}\right\}$, then $\sum_{i=1}^{n} \alpha_{i} a_{i}=b$ for $\alpha=\left(\alpha_{1}, \ldots, \alpha_{n}\right) \in \Sigma_{n}$, that is, $A \alpha=b$. Then, by Theorem 3.2

$$
\alpha_{j}=\frac{\alpha_{1 j}^{m} b_{1}+\cdots+\alpha_{m j}^{m} b_{m}}{\Delta_{m} A}
$$

is the solution of the system $A \alpha=b$. Impose $\sum_{j=1}^{n} a_{j}=1$ using linearity by rows, it follows

$$
\Delta_{m} A=b_{1}\left|\begin{array}{ccc}
1 & \ldots & 1 \\
a_{21} & \ldots & a_{2 n} \\
\ldots & \ldots & \ldots \\
a_{m 1} & \ldots & a_{m n}
\end{array}\right|+\cdots+b_{m}\left|\begin{array}{ccc}
a_{11} & \ldots & a_{1 n} \\
\ldots & \ldots & \ldots \\
a_{m-1,1} & \ldots & a_{m-1 n} \\
1 & \ldots & 1
\end{array}\right|
$$

and so the (i). By $\alpha_{j} \geq 0, j=1, \ldots, n$, we obtain the (ii).

If $\alpha_{r}$ is null in the solution of the system $A \alpha=b$, then $b \in c o\left(a_{1}, \ldots, a_{r-1}, a_{r+1}, \ldots, a_{n}\right)$. As a special case, if only $\alpha_{i}, \alpha_{j}$ are nonnull in the solution of $A \alpha=b$ and $a_{i}, a_{j}$ are extreme points, then $b$ is an extreme point of the convex hull.

Example $3.3 A$ vector $b$ is in $\in \operatorname{co}\left\{a_{1}, a_{2}, a_{3}\right\}$, with $a_{i} \in \mathfrak{R}^{2}, a_{i} \neq 0, \quad r(A)=2$, if it satisfies the following conditions

$$
\frac{1}{\Delta_{2} A}\left(b_{1} \alpha_{1 j}^{2}+b_{2} \alpha_{2 j}^{2}\right) \geq 0 \quad j=1,2,3
$$

$$
\left|\begin{array}{cccc}
1 & 1 & 1 & 1 \\
b_{1} & a_{11} & a_{12} & a_{13} \\
b_{2} & a_{21} & a_{22} & a_{23}
\end{array}\right|=0
$$

Given any matrix $A$, a matrix $A^{(2)}$ such that $A^{(2)} A A^{(2)}=A^{(2)}$ is called a (2)-inverse of $A$. Let $A \in C^{m \times n}$ and $r(A)=r$. Then $\left(\Delta_{r} A\right)^{-1} A^{a d}$ is the set of (2)-inverses of $A$ iff $r\left(A^{a d}\right) \leq r$, see [9].

A (2)-inverse $A_{R N}^{(2)}$ with prescribed range $R$ and null space $N$ is the matrix $\left(\Delta_{r} A\right)^{-1} A^{a d}$ such that

(i). $\quad\left(\Delta_{r} A\right)^{-1} A^{a d} A x=x \quad x \in R$

(ii). $\quad\left(\Delta_{r} A\right)^{-1} A^{a d} y=0 \quad y \in N$

By the next proposition, a matrix $A^{c}$ such that $A^{c} A \alpha=\alpha$ with $\alpha \in \Sigma_{n}$ is obtained.

Theorem 3.4 Let $A \in C^{m \times n}$ and $r(A)=m$. The matrix $A^{c}$ is given by

$$
A^{c}=\alpha(A \alpha)^{+}+Q\left(I_{m}-(A \alpha)(A \alpha)^{+}\right) \quad Q \in C^{m \times n}, Q \neq 0 .
$$

where $(A \alpha)^{+}$denotes the Moore-Penrose inverse of A $\alpha$.

Proof. It is known that the matrix equation $X B=C$ has a solution iff $C B^{+} B=C$, in which case the general solution is $X=C B^{+}+Q\left(I-B B^{+}\right)$, where $Q$ is an arbitrary matrix of appropriate order. Impose, in the 
equation, $B=A \alpha$ and $C=\alpha$, since $(A \alpha)^{+} A \alpha=1$ the equation has the general solution

$$
A^{c}=\alpha(A \alpha)^{+}+Q\left(I_{m}-A \alpha(A \alpha)^{+}\right) \quad Q \in C^{m \times n}, Q \neq 0 .
$$

Theorem 3.5 Let co $\left(a_{1}, \ldots, a_{n}\right)$ be a convex hull. The following four statements are equivalent

(a). $b \in \operatorname{co}\left(a_{1}, \ldots, a_{n}\right)$,

(b). the linear system $A \alpha=b$ has the solution $\alpha \in \Sigma_{n}$,

(c). $\operatorname{co}(A, b)=\operatorname{co}(A)$,

(d). $A^{c} b=\alpha$.

Proof. It is immediate that (a) and (b) are equivalent. Let us show that (b) and (c) are equivalent. Suppose $b \in \operatorname{co}(A, b)=\operatorname{co}(A)$, then $b=\alpha_{1} a_{1}+, \cdots,+\alpha_{n} a_{n} \quad, \quad$ so $A \alpha=b$ with $\alpha \in \Sigma_{n}$. Conversely, suppose $A \alpha=b$ with $\alpha \in \Sigma_{n}$, that is $b=\alpha_{1} a_{1}+, \cdots,+\alpha_{n} a_{n}$ and $b \in \operatorname{co}(A)$, but it also $b=\alpha_{1} a_{1}+\cdots+\alpha_{n} a_{n}+0 b$ where $(\alpha, 0) \in \Sigma_{n+1}$, then $b \in \operatorname{co}(A, b)$. Moreover $\forall k \in \operatorname{co}(A, b)$ is

$$
\begin{aligned}
k & =\delta_{1} a_{1}+\cdots+\delta_{n} a_{n}+\delta_{n+1} b \\
& =\delta_{1} a_{1}+\cdots+\delta_{n} a_{n}+\delta_{n+1}\left(\alpha_{1} a_{1}+\cdots+\alpha_{n} a_{n}\right) \\
& =\left(\delta_{1}+\delta_{n+1} \alpha_{1}\right) a_{1}+\cdots+\left(\delta_{n}+\delta_{n+1} \alpha_{n}\right) a_{n}
\end{aligned}
$$

since

$$
\begin{aligned}
\left(\delta_{1}+\delta_{n+1} \alpha_{1}\right)+\cdots+\delta_{n}+\delta_{n+1} \alpha_{n} & =\left(\delta_{1}+\cdots+\delta_{n}\right)+\delta_{n+1}\left(\alpha_{1}+\cdots+\alpha_{n}\right) \\
& =\delta_{1}+\cdots+\delta_{n}+\delta_{n+1} \\
& =1
\end{aligned}
$$

and $\left(\delta_{i}+\delta_{n+1}+\alpha_{i}\right), i=1, \ldots, n$, are nonnegative, then $k \in \operatorname{co}(A)$.

(b) $\rightarrow$ (d) . Let $A \alpha=b, \alpha \in \Sigma_{n}$, then there exists $A^{c}$ such that $A^{c} A \alpha=\alpha$, so $A^{c} b=\alpha$. Conversely, the relation $A^{c} b=\alpha$ is satisfied by $b=A \alpha$, then there exists $b=A \alpha \in \operatorname{co}\left(a_{1}, \ldots, a_{n}\right)$.

Example 3.4 Consider the convex hull $\operatorname{co}\left(a_{1}, a_{2}, a_{3}\right), a_{i} \in \mathfrak{R}^{2}, a_{i} \neq 0$ and let

$$
\alpha=\left(\frac{\lambda_{1}}{\lambda_{1}+\lambda_{2}+\lambda_{3}}, \frac{\lambda_{2}}{\lambda_{1}+\lambda_{2}+\lambda_{3}}, \frac{\lambda_{3}}{\lambda_{1}+\lambda_{2}+\lambda_{3}}\right) \in \Sigma_{3} \text {, then }
$$

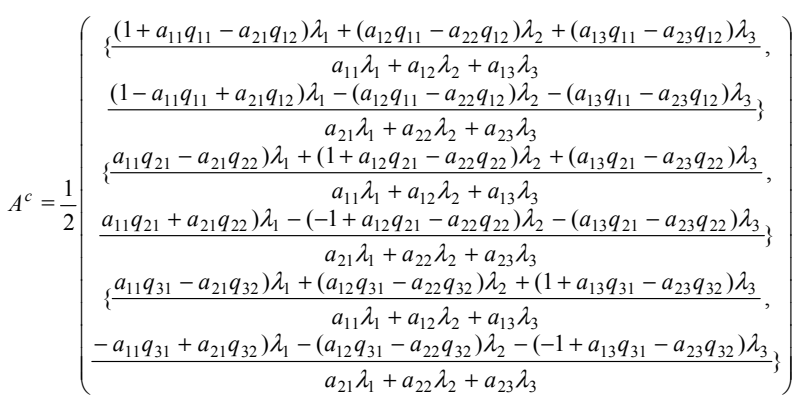

the condition $A^{c} b=\alpha$ implies for $b=\left(b_{1}, b_{2}\right)$

$$
b=\left(\frac{a_{11} \lambda_{1}+a_{12} \lambda_{2}+a_{13} \lambda_{3}}{\lambda_{1}+\lambda_{2}+\lambda_{3}}, \frac{a_{21} \lambda_{1}+a_{22} \lambda_{2}+a_{23} \lambda_{3}}{\lambda_{1}+\lambda_{2}+\lambda_{3}}\right)
$$

so

$$
\operatorname{co}\left(a_{1}, a_{2}, a_{3}\right)=\left\{b: \lambda_{i} \in \mathfrak{R}^{+}\right\}
$$

\section{Symmetric Two Persons Games}

Denote by $\Gamma_{2}=(X, X, \Phi)$ the triplet of a two persons infinite, symmetric game in normal form, with $X$ the full strategy space, see [7] and [8]. $X=c o S$ is the convex hull of subset $S$ of Euclidean n-dimensional linear space $\mathfrak{R}^{n}$, with the null vector $\underline{0} \in S$, and let $\Phi: X \times X \rightarrow \mathfrak{R}$ be the bilinear, skewsymmetric, convex payoff function.

Explicitly, impose the following axioms

1) If $x_{1} \lambda_{1}+\cdots+x_{n} \lambda_{n}=x \in X$, where $x_{i} \in X$ and $\lambda_{i} \geq 0, \quad \sum_{i=1}^{n} \lambda_{i}=1$, then

$$
\Phi(x, y)=\Phi\left(\sum_{i=1}^{n} \lambda_{i} x_{i}, y\right)=\sum_{i=1}^{n} \lambda_{i} \Phi\left(x_{i}, y\right) \quad y \in X
$$

likewise, if $y_{1} \delta_{1}+\cdots+y_{n} \delta_{n}=y \in Y$, where $y_{i} \in X$ and $\delta_{i} \geq 0, \quad \sum_{i=1}^{n} \delta_{i}=1$, then

$$
\Phi(x, y)=\Phi\left(x, \sum_{i=1}^{n} \delta_{i} y_{i}\right)=\sum_{i=1}^{n} \delta_{i} \Phi\left(x, y_{i}\right) \quad x \in X
$$

2) For every $x, y \in X \times X$, it holds $\Phi(x, y)=-\Phi(y, x)$

The payoff function $\Phi: X \times X \rightarrow \Re$ satisfies the following properties

a) $\forall x \in X$, by the second axiom, $\Phi(x, x)=-\Phi(x, x)$, that is $\Phi(x, x)=0$.

b) $\forall x, y \in X, 0<\alpha<1$, since $X$ is convex, $\alpha x+(1-\alpha) \underline{0}=\alpha x \in X$. Moreover

$\Phi(\alpha x, y)=\Phi(\alpha x+(1-\alpha) \underline{0}, y)=\alpha \Phi(x, y)+(1-\alpha) \Phi(\underline{0}, y)$ that is

$$
\Phi(\alpha x, y)=\alpha \Phi(x, y)+(1-\alpha) \Phi(\underline{0}, y)
$$


by $x=y$, it follows

$$
\Phi(\alpha y, y)=(1-\alpha) \Phi(\underline{0}, y)
$$

comparing (8) and (9), it follows

$$
\alpha \Phi(x, y)=\Phi(\alpha x, y)-\Phi(\alpha y, y)
$$

In the same way of theorem 2.1, the analytic form of the payoff $\Phi$ is now obtained.

For any two vectors $a_{1}, a_{2} \in X$ let

$a_{i}=n\left\|a_{i}\right\| \sum_{j=1}^{n} \frac{1}{n} \frac{a_{j i}}{\left\|a_{i}\right\|} x_{j}=n\left\|a_{i}\right\|\left(\frac{1}{n\left\|a_{i}\right\|} x_{1}+\cdot \cdot+\frac{1}{n} \frac{a_{n i}}{\left\|a_{i}\right\|} x_{n}\right)=n\left\|a_{i}\right\| b_{i}$

then, by the proposition 2.3 , it follows

$\Phi\left(a_{1}, a_{2}\right)=\Phi\left(n\left\|a_{1}\right\| b_{1}, n\left\|a_{2}\right\| b_{2}\right)$

$=n^{2}\left\|a_{1}\right\|\left\|a_{2}\right\| \Phi\left(b_{1}, b_{2}\right)+n\left\|a_{1}\right\|\left(1-n\left\|a_{2}\right\| \Phi \Phi\left(b_{1}, \underline{0}\right)\right.$

$+n\left\|a_{2}\right\|\left(1-n\left\|a_{1}\right\|\right) \Phi\left(\underline{0}, b_{2}\right)+\left(1-n\left\|a_{1}\right\|\right)\left(1-n\left\|a_{2}\right\|\right) \Phi(\underline{0}, \underline{0})$

using convex linearity

$$
\begin{aligned}
& \Phi\left(a_{1}, a_{2}\right)=n^{2}\left\|a_{1}\right\|\left\|a_{2}\right\|\left(\frac{1}{n} \Phi\left(\frac{a_{11}}{\left\|a_{1}\right\|} x_{1}, b_{2}\right)+\cdots+\frac{1}{n} \Phi\left(\frac{a_{n 1}}{\left\|a_{1}\right\|} x_{n}, b_{2}\right)\right. \\
& +n\left\|a_{1}\right\|\left(1-n\left\|a_{2}\right\|\right)\left(\frac{1}{n} \Phi\left(\frac{a_{11}}{\left\|a_{1}\right\|} x_{1}, \underline{0}\right)+\cdots+\frac{1}{n} \Phi\left(\frac{a_{n 1}}{\left\|a_{1}\right\|} x_{n}, \underline{0}\right)\right. \\
& +n\left\|a_{2}\right\|\left(1-n\left\|a_{1}\right\|\right)\left(\frac{1}{n} \Phi\left(\underline{0}, \frac{a_{12}}{\left\|a_{2}\right\|} x_{1}\right)+\cdots+\frac{1}{n} \Phi\left(\underline{0}, \frac{a_{n 2}}{\left\|a_{2}\right\|} x_{n}\right)\right. \\
& +\left(1-n\left\|a_{1}\right\|\right)\left(1-n\left\|a_{2}\right\|\right) \Phi(\underline{0}, \underline{0}) \\
& =\left(\left\|a_{1}\right\|\left\|a_{2}\right\|\right) \sum_{i=1}^{n} \sum_{j=1}^{n} \Phi\left(\frac{a_{i 1}}{\left\|a_{1}\right\|} x_{i}, \frac{a_{j 2}}{\left\|a_{2}\right\|} x_{j}\right) \\
& +\left\|a_{1}\right\|\left(1-n\left\|a_{2}\right\|\right) \sum_{i=1}^{n} \Phi\left(\frac{a_{i 1}}{\left\|a_{1}\right\|} x_{i}, \underline{0}\right) \\
& +\left\|a_{2}\right\|\left(1-n\left\|a_{1}\right\|\right) \sum_{j=1}^{n} \Phi\left(\underline{0}, \frac{a_{j 2}}{\left\|a_{2}\right\|} x_{j}\right) \\
& +\left(1-n\left\|a_{1}\right\|\right)\left(1-n\left\|a_{2}\right\|\right) \Phi(\underline{0}, \underline{0})
\end{aligned}
$$

by the (1)

$\Phi\left(a_{1}, a_{2}\right)=\sum_{i=1}^{n} \sum_{j=1}^{n} a_{i 1} a_{j 2} \Phi\left(x_{i}, x_{j}\right)+\sum_{i=1}^{n} \sum_{j=1}^{n} a_{i 1}\left(\left\|a_{2}\right\|-a_{j 2}\right) \Phi\left(x_{i}, \underline{0}\right)$

$+\sum_{i=1}^{n} \sum_{j=1}^{n} a_{i 2}\left(\left\|a_{1}\right\|-a_{j 2}\right) \Phi\left(\underline{0}, x_{i}\right)+\sum_{i=1}^{n} \sum_{j=1}^{n}\left(\left\|a_{1}\right\|-a_{i 1}\right)\left(\left\|a_{2}\right\|-a_{j 2}\right) \Phi(\underline{0}, \underline{0})$

$+\sum_{i=1}^{n} a_{i 1}\left(1-n\left\|a_{2}\right\|\right) \Phi\left(x_{i}, \underline{0}\right)+\sum_{i=1}^{n} a_{i 2}\left(1-n\left\|a_{1}\right\|\right) \Phi\left(\underline{0}, x_{i}\right)$

$+\sum_{i=1}^{n}\left(1-n\left\|a_{2}\right\|\right)\left(\left\|a_{1}\right\|-a_{i 1}\right) \Phi(\underline{0}, \underline{0})+\sum_{i=1}^{n}\left(1-n\left\|a_{1}\right\|\right)\left(\left\|a_{2}\right\|-a_{i 2}\right) \Phi(\underline{0}, \underline{0})$

$+\left(1-n\left\|a_{1}\right\|\right)\left(1-n\left\|a_{2}\right\|\right) \Phi(\underline{0}, \underline{0})$
By the symmetry of the game, $\Phi(\underline{0}, \underline{0})=0$. The real value $\Phi\left(a_{1}, a_{2}\right)$ is so determined by $\Phi\left(x_{i}, x_{j}\right)$ and $\Phi\left(x_{i}, \underline{0}\right)$. Denoting by $(\bar{x}, \bar{y})$ a solution of the game, the choice of the $\Phi\left(x_{i}, x_{j}\right)$ and $\Phi\left(x_{i}, \underline{0}\right)$ must satisfy the following inequalites

$$
\left\{\begin{array}{l}
\Phi(\bar{x}, \underline{0}) \geq \Phi\left(x_{i}, \underline{\overline{0}}\right) \geq \Phi\left(x_{i}, \bar{y}\right) \\
\Phi(\bar{x}, \underline{0}) \geq \Phi(\bar{x}, \bar{y}) \geq \Phi(\underline{0}, \bar{y}) \quad \mathrm{i}=1, \ldots, \mathrm{n}
\end{array}\right.
$$

and

$$
\left\{\begin{array}{c}
\Phi\left(\bar{x}, x_{j}\right) \geq \Phi\left(x_{i}, x_{j}\right) \geq \Phi\left(x_{i}, \bar{y}\right) \\
\Phi\left(\bar{x}, x_{j}\right) \geq \Phi(\bar{x}, \bar{y}) \geq \Phi\left(x_{i}, \bar{y}\right) \quad \mathrm{i}, \mathrm{j}=1, \ldots, \mathrm{n}
\end{array}\right.
$$

Suppose $X$ be a compact, convex set and define

$$
\max _{y}=\left\{x^{*}: x \in X \Rightarrow \Phi\left(x^{*}, y\right) \geq \Phi(x, y)\right\} \quad y \in X
$$

and

$$
\min _{x}=\left\{y^{*}: y \in X \Rightarrow \Phi\left(x, y^{*}\right) \leq \Phi(x, y)\right\} \quad x \in X
$$

then, by the imposed conditions on $\Phi$ and $X, \max _{y}$ and $\min _{x}$ are not empty and compact, convex sets.

The map $\Phi: X \times X \rightarrow\left\{\min _{x} \times \max _{y}\right\}, \forall(x, y) \in X^{2}$ is upper semicontinuous and we can use the following proposition, see [8]

Theorem 4.1 (Kakutani's fixed-point theorem) If $\Phi:(x, y) \rightarrow\left\{\min _{x} \times \max _{y}\right\}$ is an upper semicontinuous map on compact, convex $X \times X$ and $\{\Phi(x, y)\}$ is a convex set, then, at least an element $(\bar{x}, \bar{y}) \in X \times X$ exists such that $(\bar{x}, \bar{y}) \in \Phi(\bar{x}, \bar{y})$.

By the theorem 4.1, an element $(\bar{x}, \bar{y})$ exists with $\bar{y} \in \min _{x}$ and $\bar{x} \in \max _{y}$. Since $\bar{x} \in \max _{y}$ it holds $\forall x, \Phi(x, \bar{y}) \leq \Phi(\bar{x}, \bar{y})$ and $\forall y, \Phi(\bar{x}, \bar{y}) \leq \Phi(\bar{x}, y)$, then

$$
\forall x, y \in X \quad \Phi(x, \bar{y}) \leq \Phi(\bar{x}, \bar{y}) \leq \Phi(\bar{x}, y)
$$

that is, $(\bar{x}, \bar{y})$ is a solution of the game $\Gamma_{2}$.

\section{References}

[1] M.Carter Foundations of mathematical economics, Cambridge MIT PRESS, 2001.

[2] J.W.S. Cassels Measures of the non-convexity of sets and the Shapley-Folkman-Starr theorem Mathematical Proceedings of the Cambridge Philosophical Society Vol. 78 pp. 433-436, 1975.

[3] H.G. Egglestone Convexity Cambridge University press London, 1958. 
[4] C.Faith Algebra I Rings, Modules, and Categories Springer-Verlag, 1981.

[5] F.Fineschi, R.Giannetti Adjoints of a Matrix Journal of Interdisciplinary Mathematics. Vol.11, No. 1, pp.39-65. Taru Publications, 2008 .

[6] J.B.Hiriart-Urruty, C.Lemaréchal Fundamentals of Convex Analysis Springer-Verlag, 2001.

[7] T. Iimura, T. Watanabe Existence of a pure strategy equilibrium in finite symmetric games where payoff functions are integrally concave Discrete Applied Mathematics, Forthcoming 2012.
[8] S. Karlin Mathematical Methods and Theory in Games, Programming, and Economics - The Theory of Infinite Games Addison-Wesley, 1959.

[9] Y. Wey A characterization and representation of the generalized inverse $A_{R N}^{(2)}$ and its applicatations, Linear Algebra Appl. N. 280 pp 87-96, 1998.

[10] Lin Zhou A simple proof of the Shapley-Folkman theorem Economic Theory Vol.3, pp. 371-372, 1993. 